\title{
Pulmonary perfusion imaging applied to prediction of pulmonary vascular pressures in mitral and aortic valve disease
}

\author{
D N F HARRIS, D N T TAYLOR, K S RAO, N CONWAY, D M ACKERY \\ From the Wessex Regional Cardiothoracic Unit, and the Wessex Regional Department of \\ Nuclear Medicine, Southampton
}

SUMMARY Radionuclide lung perfusion imaging was performed on 27 patients with valvular disease of the left heart. The ratio of upper to total counts for the lungs, determined by computer, was correlated against pulmonary vascular mean pressures. A close correlation $(r=0.91)$ was obtained against pulmonary wedge pressure.

After corrective cardiac surgery upper/total ratios fell towards normal in four patients in whom pulmonary vascular pressures were measured and the correlation persisted.

This simple non-invasive index can be used to follow changes in pulmonary venous hypertension.

The effect of gravity on the pulmonary blood pattern is well recognised. The normal distribution which favours the dependent regions of the lung alters when pulmonary venous pressure rises in left sided heart disease such as mitral stenosis and, using a radioisotope method, this may be quantified to reflect changes in left atrial pressure. The paper correlates this alteration with pulmonary arterial and indirect left atrial pressures measured at catheterisation and shows the change in perfusion pattern after corrective cardiac surgery.

\section{Patients and methods}

Twenty-seven patients undergoing catheterisation for diagnostic reasons, aged 19 to 74 (mean 58), were studied: 20 with mitral stenosis, eight with mitral regurgitation, three with aortic stenosis, and four with aortic regurgitation. Nineteen had clinical evidence of pulmonary venous hypertension with pulmonary venous congestion on chest radiograph. Patients with clinical, radiographic, or isotopic evidence of parenchymal lung disease or pulmonary embolism were excluded before the study. Four patients were reimaged and had right-heart catheterisation repeated six weeks after corrective cardiac surgery. Twelve patients were reimaged at this point without recatheterisation.
Cardiac catheterisation was carried out under local anaesthetic, using standard techniques, after premedication with atropine $0.6 \mathrm{mg}$ and either lorazepam $2 \mathrm{mg}$ or promethazine $25 \mathrm{mg}$, and diamorphine 2.5 to $5.0 \mathrm{mg}$. We studied the mean pulmonary arterial and pulmonary wedge pressures.

Two to five days after catheterisation (mean $2 \cdot 4$ ), during which time no patient showed any clinical change, lung perfusion images were obtained by slow intravenous injection of $4 \mathrm{mCi}$ of ${ }^{99} \mathrm{~m} \mathrm{Tc}$-albumin microspheres, with the patient seated in order to maximise the perfusion gradient.

Cursors were placed across the top and bottom of the posterior lung image. A horizontal line was placed by the computer exactly half-way between the cursors and the number of counts integrated for the upper half (U), lower half (L), and total (T) of the image field. The same analysis was applied to the anterior view, but using the posterior image positions of the cursors as the reference (Fig. 1). The geometric mean of the counts from the anterior and posterior views

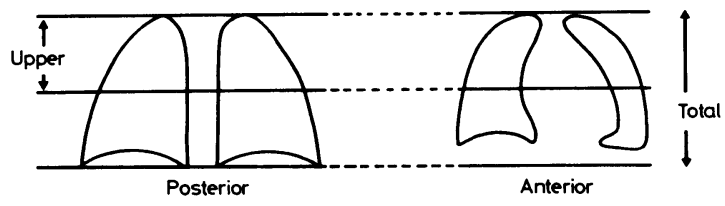

Fig. 1 Position of the cursors on posterior and anterior lung perfusion images with automatic division into upper and lower halves by the computer. 
was taken to give composite $\mathrm{U}, \mathrm{L}$, and T counts. The geometric mean is superior to the arithmetic mean ${ }^{1}$ and provides an approximately uniform counting efficiency through the lung; no correction was made for lung volume. Though only $U$ and $T$ counts were used in correlations, $\mathrm{L}$ counts were also noted to ensure that $U+L=T$, and that the number of channels in $U$ and $L$ were the same. $U / T$ was then calculated and plotted against the pulmonary vascular pressures. $\mathrm{U} / \mathrm{T}$ was chosen in place of $\mathrm{U} / \mathrm{L}$, which others have used, ${ }^{2-5}$ to minimise errors in height determination; for a given image, a change in $U$ produces an opposite change in $L$, while $T$ is unchanged. The error is thus magnified if $\mathrm{U} / \mathrm{L}$ is used.

\section{Results}

The correlations between the distribution of perfusion and mean pulmonary wedge pressure and mean pulmonary artery pressure for the 27 patients are shown in Fig. 2 and 3. The results from four patients

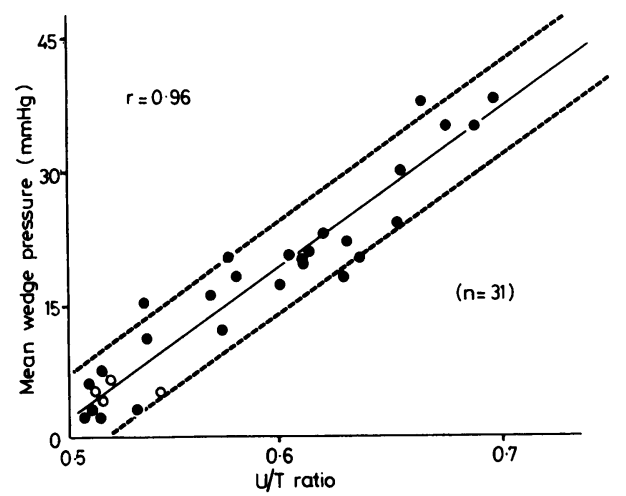

Fig. 2 Correlation between mean pulmonary wedge pressure and $U / T$ ratio. Dotted lines represent $\pm 1 S D( \pm m m H g)$. Open circles are for patients recatheterised after corrective surgery.

who were studied postoperatively are shown in Fig. 4, showing the return of the perfusion pattern to normal six weeks after operation. These points are included in Fig. 2 and 3, identified by open circles. All four patients showed a normal postoperative perfusion pattern and had a normal wedge pressure postoperatively.

Fig. 5 shows the results from the 12 patients restudied postoperatively who did not have postoperative catheterisation. The wedge pressure column shows preoperative pressures measured at catheterisation and postoperative 'pressures' estimated from the postoperative U/T ratio using the regression line of

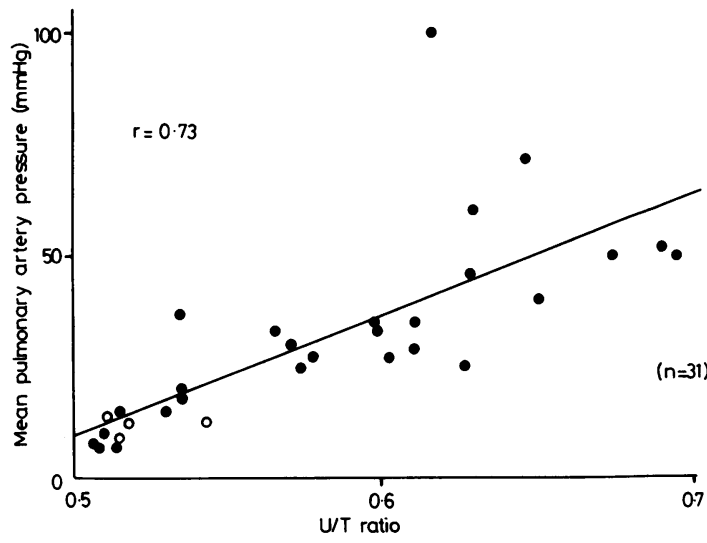

Fig. 3 Correlation between mean pulmonary artery pressure and $U / T$ ratio showing greater spread of values.

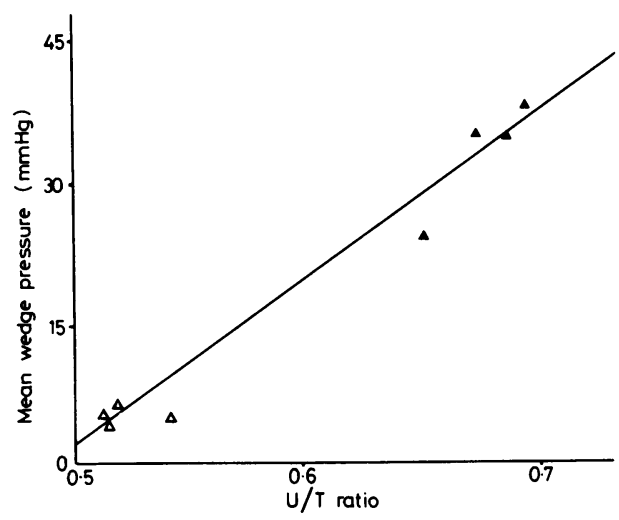

Fig. 4 Preoperative (solid triangles) and postoperative (open triangles) measurements in four patients recatheterised after corrective surgery. The regression line from Fig. 2 is superimposed.

Fig. 2. One patient with a preoperative wedge pressure of $20 \mathrm{mmHg}$ shows an estimated postoperative wedge pressure of $16 \mathrm{mmHg}$. There was, however, no clinical evidence of residual pulmonary venous hypertension.

\section{Discussion}

The mechanism causing upper lobe blood diversion in patients with raised left atrial pressure is uncertain; West et al. ${ }^{6}$ have suggested that where the hydrostatic pressure in the pulmonary venules exceeds the pulmonary capillary pressure, oedema occurs, obstructing the capillaries and reducing perfusion in the dependent zones. Initially these changes are reversible, but in time they may become permanent 


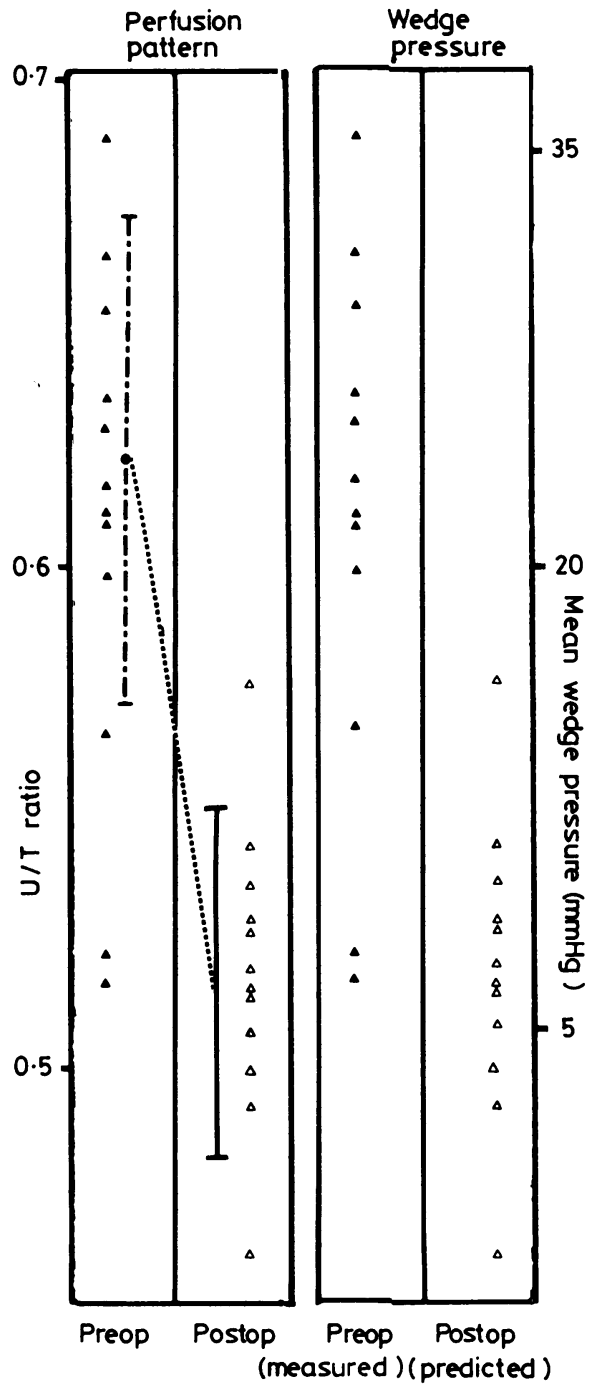

Fig. 5 Pre-and postoperative U/T ratios in 12 patients (not recatheterised) showing the fall after corrective surgery. The figures for 'wedge pressure' are devised from the measured $U / T$ ratio and the regression line of Fig. 2 and not true pressures. Vertical bars represent $\pm 1 S D$.

with hyalinisation of capillaries and venules. ${ }^{7}$

The change in the distribution of perfusion has been shown radiologically ${ }^{8}$ and using radioactive gases $^{9}$ or albumin aggregates. ${ }^{23}$ Attempts to correlate the change in the perfusion pattern with the pulmonary wedge pressure have met with varying success, with correlations of $r=0.91,{ }^{2}$ to $r=0.56 .^{5}$ Since a non-invasive index of left atrial pressure would be useful, a further investigation of the perfusion imaging, using a simple automatic technique, was considered justified. The correlation of $r=0.96$ in 27 patients with left-sided valvular disease, both aortic and mitral, equals that of Friedman and Braunwald ${ }^{2}$ who used a similar method on patients with mitral valve disease alone.

There are several possible reasons for the reported variability in correlation between the perfusion pattern and the pulmonary wedge pressure.

Focal defects in perfusion must be excluded if U/T is to represent left atrial pressure, and the gamma camera is more sensitive in the detection of such abnormalities than earlier imaging equipment such as the rectilinear scanner ${ }^{2}$ or focused detectors. ${ }^{5}$

The choice of areas to be quantified is critical. In our study and in that of Guintini et al., ${ }^{5}$ the U/L ratio was altered considerably by small changes in the position of the dividing line. We suggest that the simple automatic method presented here minimises such errors; choosing the areas by more complex methods - division into thirds, ${ }^{5}$ using a dividing crystal $^{3}$ - may account for the lower correlation achieved.

Enlargement of the left ventricle or atrium could reduce the counts in the lower half of the left lung irrespective of the height of the pressure in the left atrium; this would produce an artificially high U/T ratio. In the present series the presence or absence of cardiomegaly did not affect the correlation of $\mathrm{U} / \mathrm{T}$ against pulmonary wedge pressure, but only six of the patients had cardiothoracic ratios greater than 0.52 , and the interpretation of $\mathrm{U} / \mathrm{T}$ ratios in the presence of severe cardiomegaly may be difficult.

Variations in thoracic shape might be expected to produce a greater spread of U/T ratios for a given pulmonary wedge pressure than we observed. If the change in $U / T$ ratios between sequential measurements is used to assess the success of some intervention, any such error will be minimised. The claim that absolute pulmonary wedge pressures can be predicted from a single $U / T$ ratio cannot be made with confidence from this small series.

Although the correlation of U/T ratio against the pulmonary wedge pressure remains close at high pressures, it can be difficult to measure such pressures accurately, and the correlation we have shown cannot be taken to hold for direct left atrial pressure. Measurement of left atrial pressure in addition would be helpful and should determine the relative contributions of left atrial pressure and pulmonary resistance in determining the $U / T$ ratio.

The lower correlation of U/T against mean pulmonary arterial pressure is to be expected if, as West et al. ${ }^{6}$ suggest, it is the height of pulmonary venous pressure that determines the distribution of pulmonary perfusion. 
Several authors have shown that pulmonary venous hypertension, and therefore passive pulmonary arterial hypertension, is reversible after cardiac surgery. Braunwald et al. ${ }^{10}$ investigating patients from two to 20 months after operation showed a pronounced fall in both pressures at two months, with improvement continuing to occur up to 20 months. Dalen $e t$ al., ${ }^{11}$ studying patients with disc prostheses immediately after operation, showed that pulmonary wedge pressure fell sharply in the first 24 hours. It is not yet known whether the pattern of pulmonary blood flow reverts to normal as rapidly as the pressure change, but most of our patients showed a normal pattern six weeks after operation. It is not yet known to what extent the severity and duration of pulmonary venous hypertension preoperatively influences the rate of change in both pressure and flow pattern postoperatively. The good correlation in preoperative results, and the fall in U/T ratio in all four patients with high preoperative wedge pressures, measured postoperatively, suggests that where a fall in $\mathrm{U} / \mathrm{T}$ ratio is observed, this is likely to be accompanied by a corresponding fall in left atrial pressure. The patient with a U/T ratio which remained high postoperatively though lower than before operation, however, showed no clinical evidence of residual pulmonary venous hypertension. As the patient was not recatheterised postoperatively, it is uncertain whether this reflects a high postoperative wedge pressure or a fixed pattern of perfusion in the presence of a normal pressure.

These findings suggest that the U/T ratio measured by the method presented here is a useful non-invasive index for monitoring changes in pulmonary venous hypertension, such as occur after corrective cardiac surgery.

Care should be taken, however, in interpreting U/T ratios in patients with cardiomegaly.

\section{References}

1 Fleming JS. A technique for the absolute measurement of activity using a gamma camera and computer. Phys Med Biol 1979; 24: 176-80.
2 Friedman WF, Braunwald E. Alterations in regional pulmonary blood flow in mitral valve disease studied by radioisotope scanning. A simple nontraumatic technique for estimation of left atrial pressure. Circulation 1966; 34: 363-76.

3 Krishnamurthy GT, Srinivasan NV, Blahd WH. Pulmonary hypertension in acquired valvular cardiac disease: evaluation by a scintillation camera technique. $\mathcal{F}$ Nucl Med 1972; 13: 604-11.

4 Jebavy P, Runczik I, Oppelt A, Tilsch J, Staněk V, Widimský $\mathrm{J}$. Regional pulmonary function in patients with mitral stenosis in relation to haemodynamic data. $\mathrm{Br}$ Heart f 1970; 32: 330-6.

5 Giuntini C, Mariani M, Barsotti A, Fazio F, Santolicandro A. Factors affecting regional pulmonary blood flow in left heart valvular disease. Am $\mathcal{F}$ Med 1974; 57: 421-36.

6 West JB, Dollery CT, Heard BE. Increased pulmonary vascular resistance in the dependent zone of the isolated dog lung caused by perivascular oedema. Circ Res 1965; 17: 191-206.

7 Olsen EGJ. Perivascular fibrosis in lungs in mitral valve disease. $\mathrm{Br} \mathcal{F}$ Dis Chest 1966; 60: 129-36.

8 Doyle AE, Goodwin AF, Harrison CV, Steiner RV. Pulmonary vascular patterns in pulmonary hypertension. Br Heart f 1957; 19: 353-65.

9 Dollery CT, West JB. Regional uptake of radioactive oxygen, carbon monoxide and carbon dioxide in the lungs of patients with mitral stenosis. Circ Res 1960; 8: 765-71.

10 Braunwald E, Braunwald NS, Ross J, et al. Effects of mitral valve replacement on the pulmonary vascular dynamics of patients with pulmonary hypertension. $N$ Engl $\mathcal{F}$ Med 1965; 273: 509-14.

11 Dalen JE, Matloff JM, Evans GL, et al. Early reduction of pulmonary vascular resistance after mitral valve replacement. $N$ Engl f Med 1967; 277: 387-94.

Requests for reprints to Dr D N F Harris, Cardiology Unit, Centre Block, General Hospital, Southampton SO9 4XY, Hants. 